BMJ Open Sport \& Exercise Medicine

\title{
Determination of cervical stenosis in rugby players using an alternative radiographic method
}

\author{
Gustavo C Bornholdt, ${ }^{1}$ Bruno Siqueira Campos Lopes, ${ }^{2}$ \\ Pedro Francisco Senne Paz, ${ }^{1}$ Arnaldo José Hernandez, ${ }^{1}$ André Pedrinelli ${ }^{1}$
}

To cite: C Bornholdt G, Siqueira Campos Lopes B Francisco Senne Paz P, et al. Determination of cervical stenosis in rugby players using an alternative radiographic method. BMJ Open Sport \& Exercise Medicine 2018:4:e000411. doi:10.1136/ bmjsem-2018-000411

Accepted 3 September 2018

\section{ABSTRACT}

Purpose To find a radiographic method that best correlates with the mean subaxial cervical space available for the cord (MSCSAC) by using a fixed size parameter as radiographic reference, in contrast to the use of vertebral bodies as reference in the mean subaxial cervical Torg ratio (MTorg).

Methods The study was approved by an institutional review board and written informed consent was obtained. Radiographs and cervical neck MRI were obtained from 18 male rugby athletes (age 18-30 years). Rheumatic disease, symptomatic cervical orthopaedic disease and previous cervical injury were used as exclusion criteria. MSCSAC and MTorg were calculated for each individual as the space available for the cord and Torg ratio averages from C3 to C6, respectively. A new radiographic method, using a metal bar as a size parameter (the corrected diameter of the cervical canal - CDCC), was also calculated for each individual, as well as its average from C3 to C6 (mean corrected diameter of the cervical canal - MCDCC). Values obtained for MCDCC and MTorg were correlated with those obtained by the MSCSAC using Pearson's coefficient.

Results Four volunteers were excluded due to previous cervical injury. In total, 14 subjects had their radiographs and MRls analysed. Pearson's correlation between MSCSAC and MTorg was $0.5706(\mathrm{p}=0.033)$. The correlation between MSCSAC and MCDCC was $0.6903(\mathrm{p}=0.006)$. Conclusion MCDCC correlates better than MTorg with MSCSAC and may be a better radiographic option than MTorg for cervical stenosis evaluation.

\section{(c) Author(s) (or their} employer(s)) 2018. Re-use permitted under CC BY-NC. No commercial re-use. See rights and permissions. Published by BMJ.

${ }^{1}$ Sports Medicine Group of the Orthopedics Institute, Hospital das Clínicas da Faculdade de Medicina da Universidade de São Paulo, Sao Paulo, Brazil ${ }^{2}$ Department of Radiology, Hospital Beneficência Portuguesa, São Paulo, Brazil

Correspondence to Dr Gustavo C Bornholdt; falaguto@gmail.com
Collision sports expose athletes to risk of cervical injuries. Although most of these lesions do not represent a major risk, serious injuries may occur. ${ }^{1-3}$ Cervical cord neurapraxia $(\mathrm{CCN})$ and stingers are injuries due, respectively, to neurapraxia of the cervical spinal cord and cervical nerve roots. ${ }^{4}$ These injuries are associated with cervical stenosis, the reduction in the sagittal diameter of the cervical canal. ${ }^{4-6}$ In addition to the risk of CCN and stingers, there are no reports of complete neurological recovery in athletes with cervical stenosis who had suffered a

\section{INTRODUCTION}

What are the new findings?

- Using a fixed-length referential (corrected diamete of the cervical canal - CDCC) to overcome radiographic magnification errors seems to be better than using vertebrae body as a referential (Torg ratio).

- CDCC may be a more precise method than Torg ratio for cervical stenosis evaluation.

How might it impact on clinical practice in the near future?

Defining normative values, mean corrected diameter of the cervical canal (MCDCC) may be the method of choice when MRI is not available.

cervical fracture dislocation. ${ }^{7}$ Spinal stenosis is therefore among the main parameters to be evaluated in the return-to-play decision in contact sports after episodes of CCN and stingers.

Determination of spinal stenosis by radiography is difficult due to magnification errors caused by different distances between X-ray's source, the subject and the film. Torg ratio (TR), which is the ratio between the sagittal diameter of the cervical canal and the anteroposterior diameter of the vertebral body obtained in a sagittal neck radiography, can be used to overcome magnification problems in the diagnosis of cervical stenosis. ${ }^{5}$ The size variability between cervical vertebrae bodies, however, may result in false-positive TR for cervical stenosis in subjects who have large vertebral bodies. ${ }^{8}$ Therefore the 0.8 cut-off value suggested for TR has good sensitivity, however with a low positive predictive value. Most recent studies suggest the space available for the cord (SAC) as a more reliable method for determination of spinal stenosis. The SAC is obtained through MRI by subtracting the diameter of the spinal cord from the diameter of the spinal canal, thus not suffering influence from vertebral body size variations. ${ }^{9}$ 

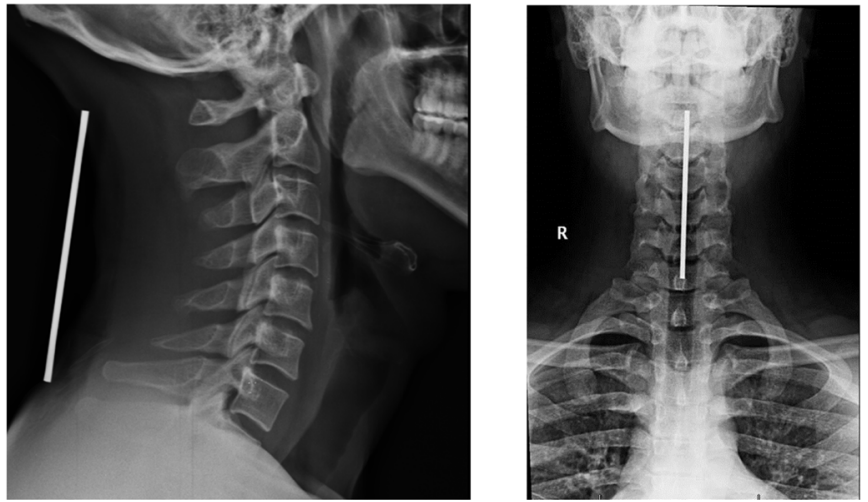

Figure 1 Cervical radiography used to determine Torg ratio and corrected diameter of the cervical canal. Anteroposterior views were used to confirm correct metal bar positioning.

The mean sagittal canal diameter for cervical levels C3C6 has been shown to be superior to isolated measures of sagittal canal, at any cervical level, in stingers prediction. The mean subaxial cervical space available for the cord (MSCSAC), which is the average SAC at the C3-C6 levels, was superior to the average Torg ratio at the C3-C6 levels (MTorg) in the prediction of stingers recurrence in NFL athletes. ${ }^{9}$ The objective of this study was to find a radiographic method that best correlates with the MSCSAC, using a fixed size parameter as radiographic reference, in contrast to MTorg's use of the vertebral body.

\section{METHODS}

An invitation to participate in the study was sent to male rugby club's players from São Paulo, Brazil, between 18 and 30 years old. All the subjects who responded to the invitation were prospectively assessed with cervical radiography and MRI. Rheumatic disease, symptomatic cervical orthopaedic disease and previous cervical injury were used as exclusion criteria. All volunteers signed an informed consent.

Cervical spine radiographs were taken from front and sagittal incidences. Cervical MRIs were obtained at T2 for evaluation at mid-sagittal view. These image studies were done in three different scheduled dates.

Lateral radiographs of the cervical spine were performed in the standing position with the central ray horizontally directed to C4 (level of the upper border of the thyroid cartilage). Image receptor was placed perpendicular to the central ray with its top edge at the level of the external auditory meatus.

Before performing each radiograph, a $100 \mathrm{~mm}$ metal bar was fixed by tape in the cervical midline using the $\mathrm{C} 7$ spinous process and superior spinous processes as landmarks. This bar was produced with steel using a lathe. Bar length was confirmed by a calliper. Anteroposterior incidence radiographs of the cervical spine were performed to confirm the vertical position of the metal bar. An example of radiographic studies can be seen in figure 1 .

Radiographs were used to calculate both the TR and the method proposed in this study, the corrected diameter of

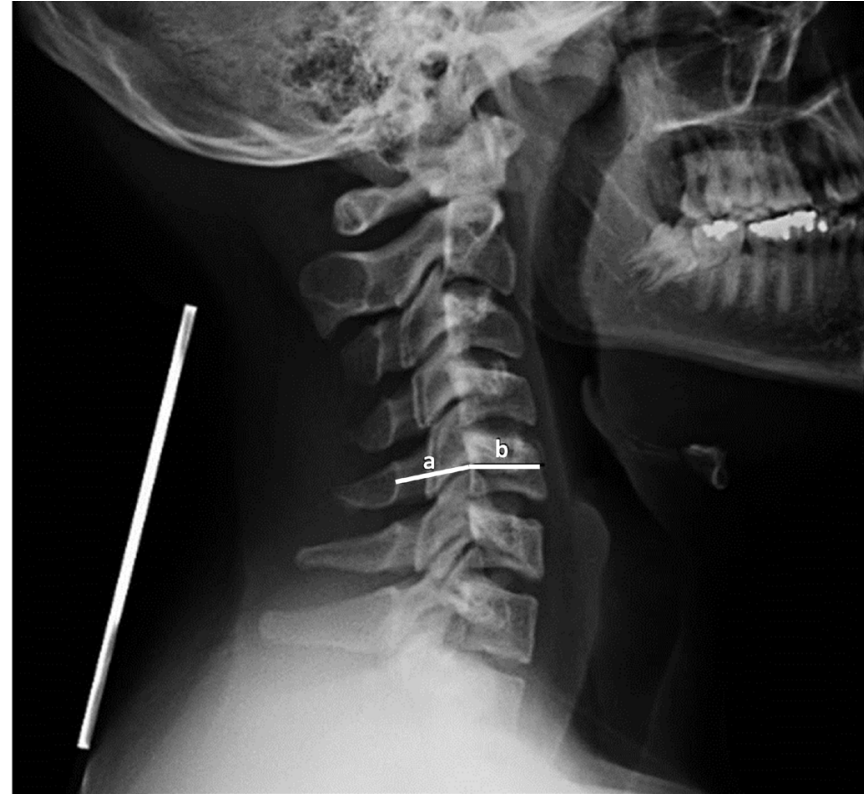

Figure 2 Torg ratio was determined by dividing the sagittal diameter of the spinal canal (a) and the anteroposterior diameter of the respective vertebral body (b).

the cervical canal (CDCC). TR was calculated by dividing the sagittal diameter of the spinal canal by the anteroposterior diameter of the vertebral body (figure 2). The sagittal diameter of the vertebral canal was established by the shortest distance from the midpoint between the superior and inferior endplates of the posterior aspect of the vertebral body, to the nearest point of the corresponding spinolaminar line. The anteroposterior diameter of the vertebral body was measured in its superior-inferior midportion.

The CDCC (figure 3) was calculated from three known variables: the sagittal diameter (in millimetres) of the cervical canal on the radiographic image, the length (in millimetres) of the metal bar image on radiography and the actual length of the metal bar $(100 \mathrm{~mm})$. With these three values, it was possible to use Thales theorem and determine the value for CDCC:

$$
\begin{aligned}
& \mathrm{CDCC} \rightarrow \mathrm{a} \\
& 100 \rightarrow \mathrm{y} \\
& \mathrm{CDCC}=100 \mathrm{a} / \mathrm{y}
\end{aligned}
$$

The averages from the C3 to C6 levels for CDCG (mean corrected diameter of the cervical canal - MCDCC) and TR (MTorg) were both calculated. A single reviewer (GCB) with experience as rugby sports physician, using the software Bit Rule, took all individual radiographic measurements. Bit Rule is a free software that can be downloaded on the internet. This software allows measurement of images on the computer screen.

MRI of the cervical spine, to calculate SAC, was performed in 1.5 Tesla unit. SAC was calculated in the mid-sagittal views of the cervical spine in $\mathrm{T} 2$ by subtracting the diameter of the spinal cord from the cervical canal diameter. The cervical canal sagittal diameter used to calculate the SAC was measured as 




Figure 3 Corrected diameter of the cervical canal values were obtained by the use of Thales theorem, with the size of the metal bar fixed over the neck as a known value, using radiography measurements of the sagittal diameter of the cervical canal (a) and bar image (y).

the shortest distance between the intervertebral disc and the corresponding spinolaminar line (figure 4). The MSCSAC was obtained by calculating the average SAC between the C3 and C6 levels. All individual MRI measurements were taken in triplicate by a single reviewer (BSCL) with experience in nervous system imaging, and the mathematical mean was calculated. The values obtained for MCDCC and MTorg were retrospectively correlated with those obtained by the

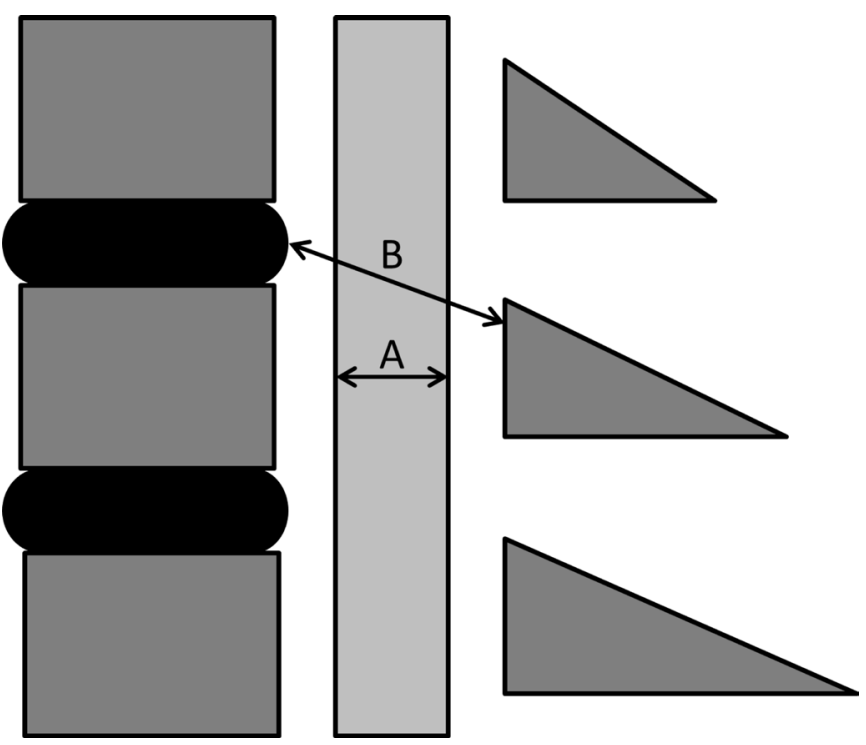

Figure 4 Space available for the cord was calculated by subtracting the mid-sagittal spinal cord diameter $(A)$ from the mid-sagittal diameter of the cervical canal at the level of the intervertebral disc (B).
Table 1 Cervical canal sample averages according to different methods

\begin{tabular}{llll}
\hline & TR & SAC $(\mathbf{m m})$ & CDCC $(\mathbf{m m})$ \\
\hline C3 & 1.02 & 7.15 & 15.95 \\
C4 & 1.00 & 6.56 & 15.52 \\
C5 & 1.01 & 6.41 & 15.54 \\
C6 & 0.94 & 6.23 & 15.51 \\
C3-C6 & 1.00 & 6.59 & 15.63 \\
\hline
\end{tabular}

CDCC, corrected diameter of the cervical canal; SAC, space available for the cord; TR, Torg ratio.

MSCSAC using Pearson's coefficient. Significance level of $95 \%$ was definite.

\section{RESULTS}

A sample of 18 male rugby athletes volunteered for this study. All subjects had experience in national or international rugby competitions. Four volunteers were excluded due to previous cervical injury. In total, 14 subjects had their radiographs and MRIs analysed. Subject's age ranged between 18 and 26 years old, their weight ranged between 63 and $117 \mathrm{~kg}$, and their height between 1.68 and $1.90 \mathrm{~m}$. Seven subjects were back position players and seven were forwards. All subjects were Caucasians.

Table 1 shows the cervical levels C3-C7 averages for the TR, SAC and CDCC found in the sample studied. MTorg, MSCSAC and MCDCC sample average are presented in the same table as the C3-C6 average.

Pearson's correlation between MSCSAC and MTorg was $0.5706(\mathrm{p}=0.033)$. The correlation between MSCSAC and MCDCC was better $(0.6903, \mathrm{p}=0.006)$.

\section{DISCUSSION}

\section{MCDCC has correlated better to MSCSAC than MTorg did}

This reinforces our hypothesis that the MCDCC is preferable since MTorg can provide results suggesting cervical stenosis even in cases where the diameter of the cervical canal has a normal size. These false-positives can occur due to the great variability of the size of the vertebral bodies, resulting in a low value of MTorg in athletes with large vertebral bodies and cervical canal of normal dimensions. ${ }^{8}$

\section{Vertebral body size variation and TR errors}

The influence of the size of the vertebral body on MTorg results was evaluated by Tierney et $a l^{10}$, who demonstrated that the variations of the vertebral body size are of greater importance than the actual variation in the diameter of the cervical canal sagittal diameter for determining the TR. When used in the evaluation of collision sports athletes, the cut-off value of 0.8 suggested for the TR may result in an increased number of false-positives as these athletes have bigger vertebral bodies than the general population. Even the use of lower cut-off points for the TR, as suggested by Herzog et $a l,{ }^{8}$ is not able to solve the 
problem due to the large interpersonal variability in the dimensions of vertebral bodies. ${ }^{10}$

\section{CDCC as an option to overcome errors due to vertebrae body size variation}

We presented MCDCC as a simple and inexpensive radiographic alternative, which depends only on the use of a known size metal bar when performing the radiographic imaging. The MCDCC could be used as a radiographic method complementary to MRI, or as its substitute in cases of difficult access to MRI. For this, we need further studies confirming its better correlation with the MSCSAC, and studies in athletes with and without history of CCN and recurrent stingers, so MCDCC normative values for increased risk of injury due to cervical stenosis could be established.

We hypothesised that MCDCC values below $13 \mathrm{~mm}$ should correlate with increased risk of CCN and recurrent stingers. We raise this hypothesis because the sagittal diameter of the cervical canal below $13 \mathrm{~mm}$ was defined as stenosis in a large study in cadavers. ${ }^{11}$ Moreover, the observations of our study group (unpublished data of athletes and non-athletes of both sexes, C Bornholdt, 2012) show that in cases of MSCSAC less than $4.3 \mathrm{~mm}$, the MCDCC value was below $13 \mathrm{~mm}$. The cut-off value of an MSCSAC of less than $4.3 \mathrm{~mm}$ has a high likelihood ratio (13.25) for recurring stingers. ${ }^{9}$

Because of technical issues, we could not achieve a larger sample of male rugby players for vertebrae imaging study. Our sample, however, was sufficient for a simple correlation study. Our results reinforce the logical assumption that a fixed-length referential (metal bar) is better than a variable one (vertebral body).

It was not our objective to compare measures of the vertebral sagittal canal diameter by MRI and CDCC. It is interesting to notice, however, the very strong correlation of 0.9253 between the MCDCC and the mean C3-C6 sagittal canal diameter measured by MRI. These data represent further support to our hypothesis that CDCC is a better radiographic method than TR for identifying cervical stenosis.

Despite the MCDCC's usefulness as a possibility for radiographic evaluation of cervical stenosis and as a useful tool for return-to-play decisions after cases of CCN and stingers, it does not replace MRI. MRI is still the best way to assess cervical stenosis since it is able to identify stenosis caused by soft tissue, such as disc protrusions and functional stenosis (defined as space with decreased cerebrospinal fluid (CSF) surrounding the spinal cord). Furthermore, beyond MSCSAC, MRI allows further information. MRI dynamic studies in cervical flexion and extension, in addition to neutral, may be useful to unveil the relationship of discs and vertebrae with related neural structures. Therefore, dynamic MRI allows identification of cervical stenosis otherwise missed or underestimated in static sequences. ${ }^{12}$

Confirmation, in subsequent studies, of MCDCC as a superior method than MTorg, as well as definition of the MCDCC normative values that determine increased risk of $\mathrm{CCN}$ and recurrent stingers, can make it the radiographic method of choice to complement MRI studies or replace them when MRI is not available.

Acknowledgements 'Mériellem Galvão Masseli and Lucas Gonçalves Dias helped in the MRI acquisition and in the article's format. The authors are thankful to them.

Collaborators ${ }^{1}$ Mériellem Galvão Masseli, Lucas Gonçalves Dias.

Funding The authors have not declared a specific grant for this research from any funding agency in the public, commercial or not-for-profit sectors.

Competing interests None declared.

\section{Patient consent Obtained.}

Ethics approval Approved by the Ethics Committee for Research Project Analysis of the Hospital das Clínicas, Faculty of Medicine, University of São Paulo.

Provenance and peer review Not commissioned; externally peer reviewed.

Open access This is an open access article distributed in accordance with the Creative Commons Attribution Non Commercial (CC BY-NC 4.0) license, which permits others to distribute, remix, adapt, build upon this work non-commercially, and license their derivative works on different terms, provided the original work is properly cited, appropriate credit is given, any changes made indicated, and the use is non-commercial. See: http://creativecommons.org/licenses/by-nc/4.0/

\section{REFERENCES}

1. Torg JS, Naranja RJ, Pavlov H, et al. The relationship of developmental narrowing of the cervical spinal canal to reversible and irreversible injury of the cervical spinal cord in football players. $J$ Bone Joint Surg Am 1996;78:1308-14.

2. Clancy WG, Brand RL, Bergfield JA. Upper trunk brachial plexus injuries in contact sports. Am J Sports Med 1977;5:209-16.

3. Robertson WC, Eichman PL, Clancy WG. Upper trunk brachial plexopathy in football players. JAMA 1979;241:1480-2.

4. Torg JS, Pavlov H, Genuario SE, et al. Neurapraxia of the cervical spinal cord with transient quadriplegia. J Bone Joint Surg Am 1986;68:1354-70.

5. Pavlov H, Torg JS, Robie B, et al. Cervical spinal stenosis: determination with vertebral body ratio method. Radiology 1987:164:771-5.

6. Torg JS, Corcoran TA, Thibault LE, et al. Cervical cord neurapraxia: classification, pathomechanics, morbidity, and management guidelines. J Neurosurg 1997;87:843-50.

7. Cantu RC. Stingers, transient quadriplegia, and cervical spinal stenosis: return to play criteria. Med Sci Sports Exerc 1997;29:233-5.

8. Herzog RJ, Wiens JJ, Dillingham MF, et al. Normal cervical spine morphometry and cervical spinal stenosis in asymptomatic professional football players. Plain film radiography, multiplanar computed tomography, and magnetic resonance imaging. Spine 1991;16:S178-86.

9. Presciutti SM, DeLuca P, Marchetto P, et al. Mean subaxial space available for the cord index as a novel method of measuring cervical spine geometry to predict the chronic stinger syndrome in American football players. J Neurosurg Spine 2009;11:264-71.

10. Tierney RT, Maldjian C, Mattacola CG, et al. Cervical spine stenosis measures in normal subjects. J Athl Train 2002;37:190-3.

11. Bajwa NS, Toy JO, Young EY, et al. Establishment of parameters for congenital stenosis of the cervical spine: an anatomic descriptive analysis of 1,066 cadaveric specimens. Eur Spine $J$ 2012;21:2467-74.

12. Zhang L, Zeitoun D, Rangel A, et al. Preoperative evaluation of the cervical spondylotic myelopathy with flexion-extension magnetic resonance imaging: about a prospective study of fifty patients. Spine 2011;36:E1134-9. 